\title{
ESTRUCTURALISMO - NEOESTRUCTURALISMO Y EL ANÁLISIS DEL DESARROLLO PRODUCTIVO
}

\author{
STRUCTURALISM - NEO-STRUCTURALISM AND ANALYSIS OF PRODUCTIVE \\ DEVELOPMENT
}

Shirley Benavides Vindas ${ }^{1}$

\begin{abstract}
Resumen
El presente artículo tiene como propósito fundamental explicar, a nivel teórico, las principales características del estructuralismo y del neoestructuralismo, con el fin de asumir elementos que coadyuven al análisis del desarrollo productivo cuando existe claramente identificado un centro del desenvolvimiento económico social versus una periferia deprimida y heterogénea en sus estructuras. No se busca analizar casos particulares de países o regiones, desde este enfoque.

La evolución del estructuralismo (finales de la década de los cuarenta y de los cincuenta) al neoestructuralismo (década de los ochenta) generó que se diera en el nuevo enfoque -como paradigma alternativo al ajuste neoliberal-, una superación del paradigma estructuralista original en el que se inspiraba; e incorporaba, al análisis los ciclos económicos, las asimetrías entre los centros y las periferias, los diversos efectos de la globalización por el regionalismo abierto y la desregulación; además, incluyó tres ejes relevantes: el económico, la equidad social y la autonomía nacional, aunque su base analítica continuaba siendo estructuralista. En esta nueva etapa se plantea el desarrollo desde la transformación productiva con equidad.
\end{abstract}

El artículo pretende explorar, en la teoría del enfoque estructuralista y neoestructuralista, los principales elementos de análisis que desde esta

Doi: http://dx.doi.org/10.15359/eys.21-50.5

Fecha de recepción: 03-10-2016. Fechas de reenvíos: 10-10-2016 / 22-10-216 / 22-12-2016. Fecha de aceptación: 26-12-2016. Fecha de publicación: 26-12-2016.

${ }^{1}$ PhD. Académica e investigadora del Programa Desarrollo Productivo, Competitividad y Comercio Internacional de la Escuela de Economía, Universidad Nacional, Costa Rica.Correo electrónico: shirley.benavides.vindas@una.cr

Shirley Benavides Vindas

(c) (i) $\$$ (O)

Revista Economía y Sociedad by Universidad Nacional is licensed under a CreativeCommons Reconocimiento-NoComercial- 
perspectiva, pueden facilitar el comportamiento de los sectores productivos y de la caracterización de territorios, además, proporciona la información necesaria para la toma de decisiones en el diseño y ejecución de políticas públicas, que eliminen las fallas de los mercados periféricos con respecto a los denominados centros y así impulsar un mayor nivel de desarrollo.

Palabras claves: estructuralismo; neoestructuralismo; centro, periferia; análisis de sectores productivos

\begin{abstract}
The main purpose of this article is to explain, at a theoretical level, the main characteristics of structuralism and neo-structuralism, in order to assume elements that contribute to the analysis of productive development, especially when there is a clearly identified center of social economic development versus a depressed and heterogeneous periphery in the structure. This approach does not attempt to analyze particular cases of countries or regions.
\end{abstract}

The evolution of structuralism (late 1940s and 1950s) to neo-structuralism (1980s) resulted in a new approach - an alternative paradigm to the neoliberal adjustment - overcoming the original structuralist paradigm from which it was inspired. This new approach included, in the analysis of economic cycles, asymmetries between centers and peripheries and the various effects of globalization resulting from open regionalism and deregulation. In addition, it included three relevant axes: economy, social equity and national autonomy, although its analytical base continued to be structuralist. In this new stage, development is proposed as a result of productive transformation with equity.

Based on a structuralist and neo-structuralist theoretical approach, this paper explores the main elements of analysis that, from this perspective, can facilitate the behavior of productive sectors and the characterization of territories, providing the necessary information for the decision-making process in the design and implementation of public policies that eliminate failures in peripheral markets regarding the so-called centers and thus promote a higher level of development.

Keywords: structuralism; neo-structuralism; center, periphery; analysis of productive sectors

2

Shirley Benavides Vindas

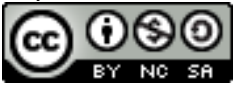

Revista Economía y Sociedad by Universidad Nacional is licensed under a CreativeCommons Reconocimiento-NoComercial- 


\section{Introducción}

Dentro de los enfoques con que cuenta la economía para realizar el análisis de las condiciones de un determinado espacio geográfico, se ha seleccionado para el presente artículo, el estructuralismo, el cual se ha aplicado en el contexto de América Latina a través de la Comisión Económica para América Latina y el Caribe (CEPAL) a partir de la década de los cuarenta y cincuenta, en un entorno periférico con un proceso de industrialización que se enfrentaba ante un deterioro en términos de intercambio y con niveles de inflación importantes.

Por su parte, el neoestructuralismo surge en el marco de la década de 1980, momento en que los países de la región latinoamericana adoptaban programas de ajuste estructural, fuertes estancamientos y una de las mayores crisis de la deuda de su historia, período que se denominó la "década perdida", como se verá en los siguientes apartados.

La corriente neoestructuralista se presentaba no solo como paradigma alternativo al ajuste neoliberal, sino también como una superación del paradigma estructuralista original, orientando su análisis de la crisis que se vivía en la región, a aspectos relacionados con lo endógeno, lo estructural que ha tenido un comportamiento histórico, y no por distorsiones que han generado la política económica o por las imperfecciones del mercado.

Este enfoque plantea cambios estructurales de la economía necesarios para facilitar el desarrollo y el crecimiento de manera inclusiva, mejorando, por un lado, la inserción en el comercio internacional de los países latinoamericanos, y por otro, reduciendo la heterogeneidad estructural, con lo que se incrementa el empleo productivo, mejorando así la distribución del ingreso.

Para el presente artículo, se realizó una exhaustiva revisión teórica e histórica primero del enfoque estructuralista y posteriormente del surgimiento de una segunda versión ajustada a un análisis mucho más ampliado, como se verá más adelante, que se denominó neoestructuralismo, dando respuesta a las condiciones de los tiempos, que planean los nuevos escenarios internacionales y regionales. El objetivo primordial para esta revisión es determinar los principales aspectos del enfoque, para su posible aplicación al estudio de los sectores productivos, quienes tienen un rol fundamental para impulsar el crecimiento y el desarrollo de las sociedades. La determinación de esto es fundamentalmente teórica, no se pretende hacer ningún estudio comparativo de tipo cuantitativo ni de generación de política pública, en el transcurso del presente artículo.

\section{El estructuralismo: sus orígenes}

En general, el estructuralismo es un enfoque filosófico que parte del valor semiótico de los procesos sociales, su propósito es el análisis de un sistema complejo, la concepción de un

Shirley Benavides Vindas

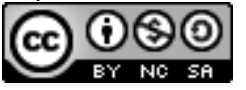

Revista Economía y Sociedad by Universidad Nacional is licensed under a CreativeCommons Reconocimiento-NoComercial- 
objeto como parte de un todo relacionado, o sea, la estructura, por lo que debe estudiarse en su conjunto y no fragmentado.

En principio las estructuras son a través de las cuales se generan un significado dentro de la cultura, mediante sus prácticas, fenómenos y actividades que dan la significación. Este enfoque ha sido utilizado como parte del estudio y análisis de una serie de enfoques para la generación de conocimiento, tales como: el marxismo con Louis Althusser; el psicoanálisis con Jacques Lancan; el filósofo y psicólogo Michael Foucault desde su crítica de la ambiciones estructurales; Claude Lévi Strauss pensador francés, desde la antropología, mediante la cual, se obtiene su mayor fundamentación y claridad, a través de los estudios de productos socioculturales con el análisis de las construcciones sociales; a Ferdinand de Saussure entre otros muchos. Todos ellos destacados exponentes en sus disciplinas que desde ella aportan de manera relacional con el estructuralismo, para la fundamentación y consolidación desde este enfoque, como se puede constatar en todos los textos especializados de dichos exponentes.

En el caso particular del marxismo, parte de su análisis se relaciona con los estudios del significado de la praxis social, en donde se detallan estructuras significativas y la construcción de los modelos lógicos, que facilitan la generación de determinados tipos de sociedades, sobre condiciones materiales y económicas específicas. También, es relevante recordar que la significación de las diferentes estructuras de la sociedad surge no solo por las prácticas sociales, sino que además se transforma dentro de la estructura misma, y da como resultados las luchas de clases, las cuales facilitaron la interpretación marxista de sus contextos.

Por su parte, la Comisión Económica para América Latina y el Caribe (CEPAL) toma este enfoque durante el año de 1949 y la década de los cincuenta, como respuesta a una serie de fenómenos económicos, sociales y políticos que se vivieron en Latinoamérica en esos años, es una revisión crítica de manera particular, relacionado con la industrialización y el proceso de cambio profundo en las estructuras económicas y sociales, por investigadores ligados en diferentes formas con la CEPAL, tales como: Raúl Prebisch, quien fue su primer precursor, Celso Furtado, Fernando Fajnzlber y José Medina, quienes dieron grandes aportes a la economía política y al pensamiento económico de la región, mediante el desarrollo de una escuela de pensamiento especialista en el estudio de las tendencias económicas y sociales de mediano y largo plazo de toda la región, desde una perspectiva estructural.

Entre otros aspectos fundamentales del estructuralismo en Latinoamérica se encuentra el desarrollo del análisis de la relación centro-periferia, en la cual, la estructura de esta última puede presentar dos facetas contrapuestas, por un lado, las homogéneas, en el sentido de que coexisten sectores donde se utilizan las técnicas avanzadas provenientes de los centros y donde la productividad del trabajo es comparable; mientras que en otras regiones se aplican técnicas obsoletas con niveles de productividad muy inferiores a los de actividades similares realizadas en las economías centrales, generando asimetrías en el desarrollo de las zonas.

4

Shirley Benavides Vindas

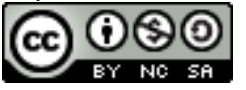

Revista Economía y Sociedad by Universidad Nacional is licensed under a CreativeCommons Reconocimiento-NoComercial- 
Las discusiones de la época se daban alrededor del concepto de subdesarrollo y las condiciones que lo generaban, buscando explicaciones y mecanismos para logar superar esa etapa y llegar a un adecuado nivel de desarrollo económico, como lo señala Octavio Rodríguez (2006):

En el aumento del bienestar material, normalmente reflejado en el alza del ingreso real por habitante, y condicionado por el incremento de la productividad del trabajo. Este incremento se considera dependiente de la adopción de métodos de producción indirectos, o sea, de métodos en los cuales se incrementa la división técnica del trabajo entre actividades, lo cual a su vez conlleva el aumento de la dotación de capital por hombre ocupado. La mayor densidad de capital se va logrando a medida que la acumulación se lleva a cabo bajo el impulso del avance tecnológico, necesario para preservar márgenes de ganancia que aseguran la continuidad de la misma.

Así pues, consideradas con el mayor nivel de abstracción, las ideas sobre el desarrollo económico coinciden con las contenidas en líneas generales en las teorías del crecimiento de origen neoclásico y keynesiano, que los conciben como un proceso de acumulación de capital estrechamente ligado al progreso técnico, mediante el cual, se logra la elevación gradual de la densidad de capital y el aumento de la productividad del trabajo y del nivel medio de vida (p. 54).

Un aspecto relevante en la discusión de la época y que posibilita un análisis más integral y robustecido es lo relacionado con la heterogeneidad estructural, la cual limitaba en las condiciones de la periferia la capacidad de generar excedentes, debido a que en esas circunstancias solo una parte poco representativa de la economía dentro de los sectores productivos, funcionan con una productividad relevante. Con todo este escenario se dan los inicios de la aplicación del enfoque estructuralista al análisis de las situaciones productivas, económicas y sociales de la época, tomando en cuenta las características centro-periferia, y los desequilibrios de la balanza de pagos, el desempleo, el deterioro de los términos de intercambio y la inflación.

\section{Estructuralismo y los sectores productivos}

Se denominan estructuralistas a quienes conceptualizan que los problemas de los países de América Latina son de origen estructural, es decir, se derivan del propio funcionamiento del sistema económico. En esta etapa se entendía que la industrialización era indispensable para lograr la convergencia que facilitaría el adecuado crecimiento. Dentro de este enfoque, el primer elemento con que se caracteriza el subdesarrollo regional, era la escasa diversidad productiva y la especialización en bienes primarios, situación que dificultaba los esfuerzos para disminuir las brechas existentes en materia de ahorro y divisas, lo que, a su vez, impone restricciones al crecimiento que además, estaba impactado por su vulnerabilidad externa.

Shirley Benavides Vindas

$$
\text { (c) (i) (5) }
$$

Revista Economía y Sociedad by Universidad Nacional is licensed under a CreativeCommons Reconocimiento-NoComercial- 
Lo anterior indica unos de los principales aspectos que componen el análisis estructural, por otra parte, Octavio Rodríguez (2006), agrega a la construcción del contenido básico del pensamiento estructuralista, las siguientes observaciones que dan un mayor panorama:

Los cambios en la dotación de los recursos, en la técnicas productivas y en la productividad del trabajo son rasgos peculiares del proceso de desarrollo a largo plazo del sistema económico; este sistema se encuentra formado por centros y periferias, los cuales difieren en cuanto a estructura y función económica, características diferenciales que constituyen el marco dentro del cual se produce el proceso de desarrollo y/o de propagación universal del progreso técnico; existen distintos ritmos del aumento de la productividad del trabajo, mayores en los centros que en la periferia; hay un nexo causal entre el deterioro de los términos de intercambio y las condiciones de estructura propias de la periferia, especialmente la tendencia a la generación continua de un excedente de mano de obra, a su vez conectada, a la diferenciación del nivel de salarios, y finalmente, el deterioro constituye un fenómeno necesario, expresión y mecanismo mediante el cual se realiza la concentración de los frutos del progreso técnico en los centros. (p. 100)

Todos los aspectos anteriores se relacionan de manera directa con el análisis del desempeño de los sectores productivos y ello refleja cómo puede impactar en el desarrollo de las regiones en las que se encuentran insertos, además, dan información para el impulso de las ventajas competitivas dinámicas que facilitan el crecimiento y posibilita una mejor distribución del ingreso, como se indicara a continuación.

En la fase inicial del pensamiento estructuralista se destacaron las limitaciones asociadas al esquema de desarrollo basado en el aprovechamiento de las ventajas comparativas estáticas las que se basan en inversiones duras, como máquinas, tecnología que sirven durante un tiempo y luego se vuelven obsoletas transformándose en barreras de salida-; además, implicaba impulsar el crecimiento de la clase trabajadora urbana y un mayor rol al empresariado industrial frente a los sectores oligárquicos agro-exportadores, de la época.

El supuesto relevante, de dicha fase, se relacionaba con la industrialización vía la sustitución de importaciones, lo cual, impulsaría las economías periféricas a lograr un crecimiento independiente y más igualitario basado en la exportación de bienes. Además, se da la diversificación y la homogeneidad en los centros. Este análisis, centro-periferia, es fundamental en el estructuralismo, su carácter es el patrón ideal de transformación de la estructura productiva, en el caso particular, de la periferia para logar una adecuada relación de intercambio, que impulse el equilibrio y crecimiento, a través del desarrollo empresarial y por ende del mejoramiento de los mercados laborales a través de mayores y mejores empleos en la periferia, con lo cual se evita las migraciones a los centros, situación que genera una serie de problemas socio económicos.

6

Shirley Benavides Vindas

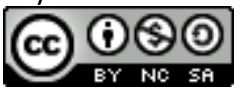

Revista Economía y Sociedad by Universidad Nacional is licensed under a CreativeCommons Reconocimiento-NoComercial- 
Surge de la transición de las economías latinoamericanas del modelo de crecimiento exportador -hacia afuera- a uno orientado a lo urbano-industrial -hacia adentro- , a partir de la perspectiva del estructuralismo, cuando la dinámica de los centros no lograba permear de manera óptima a la heterogeneidad productiva en la periferia, en la cual se generan bienes y servicios con una demanda internacional poco dinámica, y en contraste, una demanda interna creciente $y$ asimilando patrones de consumo y tecnología originaria de los centros, aspectos que se componen vigentes hoy en día en el análisis de los sectores productivos, y que continúan generando por un lado beneficios parciales, tales como un mercado laboral mayor pero con baja productividad e ingresos medios, prácticamente de subsistencia, y por otro, una concentración de la propiedad y distribución desigual del ingreso, lo cual restringe la inversión y limita el crecimiento.

Otro elemento a considerar y que es válido en la actualidad, es lo relacionado con el tejido institucional, el cual en las periferias es poco propenso a la acumulación de capital y al progreso técnico, debido a las pocas posibilidades que ofrecen los centros académicos y de formación en esas regiones, lo que no estimulaba la inversión en aquellos sectores dinámicos y que prospectan van a desarrollar, y por lo tanto, se reduce las posibilidades de una industrialización espontánea, la cual tiene un papel predominante, como lo es en la actualidad, por ser un dinamizador en el desarrollo de las regiones, ya que sin esta condición, da como resultado escenarios desiguales de crecimiento y distribución de los ingresos, lo cual a su vez, fortalece la precariedad de las condiciones de vida de los habitantes de los territorios de estudio.

Según Nora Lustig (1987), sobre este enfoque de análisis señala:

El pensamiento estructuralista considera que las características estructurales de una sociedad determinan de manera fundamental su comportamiento. Entre los factores estructurales se encuentran... la distribución del ingreso y la riqueza, los regímenes de tenencia de la tierra, el tipo y grado de especialización del comercio exterior, la densidad de las cadenas productivas, el grado de concentración de los mercados, el control de los medios de producción por distintos tipos de actores (el sector privado, el Estado y el capital transnacional), el funcionamiento de los mecanismos la penetración de innovación tecnológica, así como factores sociopolíticos asociados al grado de organización de la clases trabajadora y de otras clases de sectores influyentes, la distribución geográfica y sectorial de la población, y el nivel la calificación de esta. Para el estructuralismo estas características determinan el funcionamiento específico de los mecanismos causales y el éxito previsible de una estrategia de desarrollo.

El pensamiento estructuralista se encuadra dentro de la economía política. Ya sea en su forma más radical asociada a la tradición marxista, o en su vertiente reformista asociada a los enfoques keynesianos e institucionalistas. Para el estructuralismo no tiene sentido concebir a la sociedad como la suma de unidades familiares y

Shirley Benavides Vindas

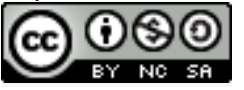

Revista Economía y Sociedad by Universidad Nacional is licensed under a CreativeCommons Reconocimiento-NoComercial- 
empresas atomizadas que toman los parámetros económicos como datos. En esta visión se reconoce que las medidas de política económica afectan el comportamiento de los actores sociales y que estos, lejos de funcionar como individuos aislados, tienden a unirse y generar grupos de presión.

Las raíces clásicas del pensamiento estructuralista se pueden encontrar en la tradición marxista/schumpeteriana en el sentido de que para el estructuralismo el sistema capitalista de libre empresa no es un sistema armónico sino inherentemente conflictivo, y su desarrollo no ocurre de manera suave, sino que se da en forma de saltos, generándose innumerables desequilibrios en el proceso mismo. (pp. 2-3)

En cuanto a la dinámica de los actores sociales en el sistema y los roles que toman en la estructura de la región en su desempeño productivo y económico, Nora Lustig (1987) agrega al respecto elementos que proceden en un análisis territorial de los sectores productivos en el contexto actual, tales como:

1. Los actores sociales más relevantes no son tomadores de precios e imprimen rigideces importantes en los mercados.

2. La relación causal no va del ahorro a la inversión sino en el sentido inverso.

3. La oferta monetaria es en general pasiva y se ajusta a la inflación y no al contrario.

4. La inversión pública es complementaria e incentiva la inversión privada.

5. El proceso de desarrollo no es ni balanceado ni armónico y se da mediante la incorporación y diseminación del progreso técnico. Este se incorpora, principalmente, a través de la nueva inversión.

6. Los bienes adquiridos esenciales desde el punto de vista tecnológico, se convierten en indispensables para sostener el crecimiento.

Como se puede observar en los principales planteamientos de la concepción estructuralista se presenta una relación directa con el comportamiento de los sectores productivos y las características de las regiones y sus elementos son relevantes para el estudio de ellos y la posible determinación de las posibilidades de impulsar su competitividad y mejorar sus niveles de desarrollo.

\section{Del estructuralismo al neoestructuralismo}

Para la década de los ochenta e inicios de los noventa, el estructuralismo evolucionó al pensamiento neoestructuralista, que se presentaba no solo como paradigma alternativo al ajuste neoliberal, sino también como una superación del paradigma estructuralista original en el que se inspiraba, esto mediante la incorporación del análisis de los ciclos económicos, las asimetrías y diversos efectos de la globalización por el regionalismo abierto y la desregulación, además, presenta tres ejes relevantes: el económico, la equidad social y la autonomía nacional, pero su base analítica continuaba siendo estructuralista.

8

Shirley Benavides Vindas

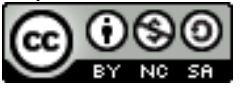

Revista Economía y Sociedad by Universidad Nacional is licensed under a CreativeCommons Reconocimiento-NoComercial- 
En la etapa neoestructuralista se plantea el desarrollo desde la transformación productiva con equidad, lo cual lo presenta mediante documentos generados desde la CEPAL en 1990. Se argumenta que la ausencia de diversidad productiva y exportadora genera una baja densidad tecnológica y un reducido encadenamiento entre los sectores productivos, lo que limita el crecimiento y sus efectos multiplicadores, debido a que las condiciones de mercado se mantenían, tales como: mano de obra abundante, que genera informalidad y pobreza. Las bajas tasas de crecimiento se explicaban por el hecho de que la institucionalidad con sistemas nacionales de innovación incipientes, falta de visión de desarrollo por parte de los estados y ausencia de inversión por parte de corporaciones globales.

Por su parte, igual que en su primera etapa, la del estructuralismo, en esta segunda, se enfoca en una macroeconomía heterodoxa con análisis que indica que la inestabilidad de precios y los niveles de actividad se han centrado en el desequilibrio externo generado por la especialización productiva y la necesidad de contar con condiciones que posibiliten un desarrollo sostenible ambientalmente, esto ante las tendencias de producción y consumo desmedido.

Por lo anterior, el neostructuralismo según la CEPAL, es un sistema abierto que se presta a establecer diálogos con otras tradiciones de pensamiento en economía, como las corrientes heterodoxas, que incluyen a los evolucionistas e institucionalistas, la escuela de la regulación, los marxistas y radicales, y los post-Keynesianos, esto debido a estas orientaciones han permitido alimentar un análisis crítico del pensamiento y las políticas económicas. Dentro del desarrollo de metodologías de análisis de la dinámica empresarial, todos estos elementos se tornan fundamentales para determinar las posibilidades de aporte real de las industrias al bienestar de las sociedades, a través de una mejor oferta en el mercado laboral, lo cual apoya la adecuada redistribución del ingreso en un país o región. Al momento de generar la estrategia de transformación productiva hacia la periferia, dentro del diseño de las políticas públicas, se debe de tomar en cuenta la serie de variables que conforman el estructuralismo y el neoestructuralismo, para impulsar la creación de empleos dignos, evitando pronunciar las desigualdades en la distribución de los ingresos y potencializando las exportaciones de las regiones que se encuentran en proceso de dinamización. Facilitando una estrategia de desarrollo "desde adentro", mediante el cual, el sector empresarial aporta en productividad, calidad, flexibilidad en la toma de decisiones, y aportes a los esfuerzos de innovación de los sectores productivos, que además respetan los recursos naturales y humanos que utiliza de manera racional.

En la propuesta neoestructuralista, el Estado, no sustituye las fuerzas del mercado con una acción excesiva sino selectiva que estimule la actividad productiva del mercado, o sea, es contar con un Estado, con una institucionalidad más eficiente que logre fortalecer los mercados y hacerlos más equitativos, aspecto fundamental del neoestructuralismo, razón por la cual las políticas públicas son fundamentales desde su diseño hasta su evaluación, y deben de tener claro su sentido de responsabilidad colectiva, respetando los recursos naturales, el Estado que

Shirley Benavides Vindas 
permita superar las fallas de mercado que se han venido produciendo en determinados mercados, para facilitar avanzar hacia una verdadera competitividad empresarial y regional, demostrando que posee capacidad de gestión y de concertación y diálogo transparente con el sector privado.

La institucionalidad pública desde los neoestructuralistas, debe de fortalecer la competitividad exterior, para lo cual, se debe en un mediano plazo, facilitar la inserción en los mercados internacionales, facilitado por mejores plataformas educacionales, innovaciones tecnológicas y aumentos de la productividad, ya que existía poca diversidad de la estructura productiva, con especialización en bienes primarios de bajo valor agregado y poco dinamismo internacional, escasos encadenamientos productivos internos y una capacidad insuficiente de generación de externalidades positivas para el crecimiento.

Según Antonio Vázquez (2000):

Las empresas toman sus decisiones de innovación en un entorno cada vez más competido y globalizado y es, precisamente, el esfuerzo por aumentar la rentabilidad de sus inversiones y ampliar la presencia en los mercados lo que constituye uno de los mecanismos claves del proceso de innovación. Desde la perspectiva del desarrollo competitivo de las economías, las innovaciones y las nuevas tecnologías no surgen fuera del sistema económico, sino que son endógenos al sistema productivo, a la economía y a la propia sociedad. (p. 5)

La CEPAL-UNESCO dio la base fundamental del neoestructuralismo, a través de su publicación "Educación y conocimiento: eje de la transformación productiva con equidad" (1992), después de un replanteamiento del desarrollo de la región latinoamericana. La igualdad con enfoque de derechos supone tres grandes desafíos:

i) lograr un crecimiento sostenido a tasas elevadas, suficientes para cerrar brechas estructurales y generar empleos de calidad;

ii) cambiar los patrones de consumo y producción en el contexto de una verdadera revolución tecnológica con sostenibilidad ambiental mediante políticas industriales y mejor gobernanza de los recursos naturales, y

iii) garantizar una mejor distribución de los aumentos de productividad mediante políticas fiscales y sociales redistributivas, una mejor repartición de los beneficios entre el capital y la mano de obra, el aumento de las capacidades y el establecimiento de sistemas de protección social universal. Las principales preocupaciones de América Latina y el Caribe respecto de la agenda de desarrollo son los retos persistentes de erradicar la pobreza y reducir la desigualdad.

10

Shirley Benavides Vindas

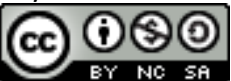

Revista Economía y Sociedad by Universidad Nacional is licensed under a CreativeCommons Reconocimiento-NoComercial- 
Uno de los elementos fundamentales para generar condiciones favorables para el desarrollo adecuado de los sectores productivos y que dentro de los objetivos de la nueva propuesta de CEPAL-UNESCO (1992) se encuentra con el surgimiento del Modelo Neoestructuralista, es la estrategia de contribuir a crear condiciones educacionales y generación de conocimientos, para la incorporación del progreso científico-tecnológico en la transformación de las estructuras productivas en un marco de equidad social. Además, dicho texto agrega que la orientación de la estrategia es la siguiente:

Los cambios que se proponen se orientan en las siguientes direcciones principales: i) desde el punto de vista político, se trata de asumir las actividades de producción y difusión de conocimientos como tareas estratégicas de largo plazo que requieren el más amplio consenso posible entre los diferentes actores sociales, y un compromiso financiero estable con su desarrollo; ii) desde el punto de vista de los contenidos, de focalizar la acción en los resultados de la educación, la capacitación y la ciencia y tecnología, y en su articulación con las exigencias del desempeño de las personas, las empresas y las instituciones en los diferentes ámbitos de la sociedad; iii) desde el punto de vista institucional, de romper el aislamiento de los establecimientos educativos y de generación y transmisión de conocimientos, e introducir modalidades de acción en que los actores tengan mayores márgenes de autonomía en las decisiones, así como mayor responsabilidad por los resultados. (CEPALUNESCO, 1992, p. 19)

Por otra parte, como complemento a lo anterior, Octavio Cortés (2011), señala que:

Las primeras propuestas de transformación productiva con equidad estaban encaminadas a retomar el camino de la industrialización de la región. Una industrialización que insertase a dicha región en la competencia internacional, pero con una clara manifestación a favor de la protección, el rechazo contundente a la apertura externa indiscriminada y una crítica a la propuesta de formación de patrones de especialización basados en la abundancia de recursos naturales. Se avanza en el concepto clave para analizar la incorporación del progreso técnico, que es "núcleo endógeno de dinamización tecnológica". El progreso técnico deja de ser una variable exógena incorporada a los bienes de capital (...)

Más aún la transformación productiva con elevado crecimiento de la productividad del trabajo tiene que acompañarse de mayores remuneraciones a los trabajadores, como un elemento importante tanto en la equidad distributiva como para la realización de la producción, de manera que la equidad se constituye en requisito de la eficiencia y la dinámica industrializadora. (pp. 61-62)

La meta dentro de esta estrategia de desarrollo que se orienta al crecimiento y la equidad, se convierte es una opción más, para orientar el análisis de los sectores productivos y su

Shirley Benavides Vindas

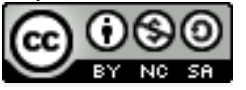

Revista Economía y Sociedad by Universidad Nacional is licensed under a CreativeCommons Reconocimiento-NoComercial- 
desempeño, tomando en cuenta las condiciones generadas desde la periferia y los centros, lo cual se complementa, con un estudio de la economía regional.

Los enfoques estudiados, estructuralismo y neoestructuralismo, brindan una guía metodológica e instrumental a considerar, que desde su perspectiva, facilitan la determinación de escenarios estructurales que dinamizan o no el desarrollo productivo; para lo cual, le asigna un papel trascendental a la heterogeneidad estructural, la cual toma en cuenta, entre otros aspectos, la heterogeneidad de: los mercados externos; las etapas del ciclo económico (diferente respuesta de los mercados en las fases recesivas y de auge); las distintas capacidades de respuesta ante los estímulos que tienen las regiones a través de su parque empresarial a los segmentos de mercados; la absorción de las tecnologías, la manera de aumentar la productividad, los grados de movilidad de los recursos y de flexibilidad de los precios, los procesos de ajuste macroeconómicos, y las percepciones o expectativas de los diversos agentes económicos, a través de los patrones distributivos y la demanda, la estructura de los sectores productivos.

Adicionado a lo anterior, se debe incluir al análisis y comprensión de la dinámica productiva, las estructuras sociales, que la afectan de manera directa, como parte de un sistema económicosocial al que pertenecen y que condicionan al desarrollo económico, esto a través de los diversos actores sociales e institucionales, que a su vez orientan ciertos comportamientos e intereses, mediante los cuales, facilitan la dinamización de los sectores productivos, a través de establecer patrones específicos de racionalidad económica.

El conjunto de todos los elementos brindados por el estructuralismo y particularmente el neoestructuralismo que amplía la zona de análisis, como ya se ha expuesto anteriormente, se convierte en parámetros que brindan un bagaje importante al momento de determinar marcos teóricos de referencia, que se complementan, para el análisis del desenvolvimiento de los sectores productivos, su competitividad y su desempeño en los mercados internacionales.

\section{Elementos para análisis de los sectores productivos desde el enfoque neo y estructuralista}

Cuando se toma un enfoque de análisis desde la estructura económica de un territorio o región determinada, todo lo expuesto supra presenta una serie de elementos que favorecen una mayor profundidad del desempeño de los sectores productivos, tales como:

Las condiciones que caracterizan tanto el centro como la periferia y la relación de intercambio entre sí, es todo un tema de análisis para determinar la competitividad y por ende las posibilidades de desarrollo de las regiones que se encuentran en la periferia, la cual en principio está en condiciones menos favorables frente a las alternativas de intercambio con el centro. Cabe referencia resaltar que a nivel del planteamiento neoestructuralista se hacía referencia al centro como los países desarrollados y a la periferia, los subdesarrollados; esta concepción

12

Shirley Benavides Vindas

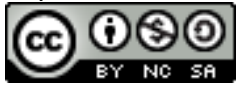

Revista Economía y Sociedad by Universidad Nacional is licensed under a CreativeCommons Reconocimiento-NoComercial- 
puede ser válida, cuando se analizan regiones desarrolladas como los centros y aquellas donde se dan una serie de asimetrías que los ubica como deprimidos.

Los principales planteamientos de la concepción estructuralista, presentan una relación directa con el comportamiento de los sectores productivos, las características de los territorios y, sus elementos relevantes para el estudio de ellos, con el propósito de determinar las posibilidades reales para impulsar su competitividad y mejorar sus niveles de desarrollo.

La determinación de la productividad del trabajo y el impacto de la técnica en la mano de obra, a través de los bienes esenciales desde el punto de vista tecnológico, se convierten en indispensables para sostener el crecimiento. Como parte fundamental para contar con las condiciones necesarias para hacerle frente a la dinámica del mercado en el cual se encuentran insertas las relaciones comerciales entre las regiones, con lo cual, se busca el aumento del bienestar, normalmente reflejado en el alza del ingreso real por habitante, y condicionado por el incremento de la productividad del trabajo.

Al evolucionar al neoestructuralismo, se incluye la incorporación del análisis de los ciclos económicos, las asimetrías y diversos efectos de la globalización por el regionalismo abierto y la desregulación, además, se dan tres ejes relevantes: el económico, la equidad social y la autonomía nacional, aunque su base analítica continuaba siendo estructuralista.

La importancia relativa que da el neoestructuralismo a través de una estrategia de transformación productiva hacia la periferia, mediante el diseño de las políticas públicas, para evitar que las desigualdades se pronuncien más a nivel de la distribución de los ingresos y la potencialización de las exportaciones, facilitando una estrategia de desarrollo "desde adentro", mediante el cual, el sector empresarial aporta en productividad y bienestar.

A mediano plazo, se debe facilitar la inserción en los mercados internacionales, mediante mejores plataformas educacionales, innovaciones tecnológicas y aumentos de la productividad, para impactar positivamente la diversidad de la estructura productiva, y los encadenamientos productivos para generar externalidades positivas para el crecimiento, todo ello fortalecido por los esfuerzos de innovación de los conglomerados empresariales.

Se debe demostrar que el Estado posee capacidad de gestión y de concertación y dialogo transparente con el sector privado, en pro de generar plataformas que faciliten la dinamización empresarial, para mejorar las condiciones sociales y económicas de las sociedades y regiones.

La institucionalidad pública desde los neoestructuralistas, debe de fortalecer la competitividad exterior, para lo cual, se debe a un mediano plazo, facilitar la inserción en los mercados internacionales, facilitado por mejores plataformas educacionales, innovaciones tecnológicas y aumentos de la productividad.

Shirley Benavides Vindas

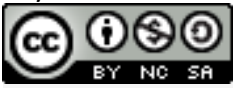

Revista Economía y Sociedad by Universidad Nacional is licensed under a CreativeCommons Reconocimiento-NoComercial- 
Es importante tener en cuenta estrategias que generan un crecimiento sostenido suficiente para cerrar brechas estructurales y generar empleos de calidad; cambiar los patrones de consumo y producción en el contexto de una verdadera revolución tecnológica con sostenibilidad ambiental mediante políticas industriales, y garantizar aumentos de productividad impulsados por las políticas fiscales y sociales redistributivas.

Para el análisis de los sectores productivos se debe de considerar, en este enfoque, su estructura; la heterogeneidad de los mercados externos; los ciclos económicos; las capacidades de respuesta que tienen las regiones a través de sus empresas ante sus mercados; las curva de aprendizaje de las tecnologías para aumentar la productividad, los grados de movilidad de los recursos y de flexibilidad de los precios; la capacidad macroeconómica; las percepciones o expectativas de los diversos agentes económicos a través de los patrones distributivos y la demanda.

Valorar los aspectos sociales, como parte del complemento del análisis de los sectores productivos, como lo han indicado otras teorías de estudio de la competitividad de manera más sistémica a través de los niveles meta.

Seguro es importante incluir otros aspectos más a nivel macroeconómico que el enfoque en mención no le dio la relevancia necesaria en su momento, tales como: las variables macroeconómicas de corto plazo, que no se incluyeron con el detalle que se requería, tanto a nivel de déficit fiscal y la liquidez monetaria, además, no se llevó a cabo un análisis las políticas de mediano plazo que orientaban a los objetivos nacionales y a su vez el desarrollo y la planificación de la región; aspectos a considerar en la actualidad, al tomar este enfoque como base para el análisis de sectores productivos y los territorios, y así poder contar con la información necesaria para la toma de decisiones y el diseño y ejecución de la política pública que busca mejores y mayores niveles de desarrollo.

\section{Referencias}

CEPAL-UNESCO (1992). Educación y conocimiento: eje de la transformación productiva con equidad. Recuperado de http://repositorio.cepal.org/bitstream/handle/11362/2130/S9250755 es.pdf?sequenc $\underline{\mathrm{e}=1}$

Cortés, O. (2011). Estructuralismo, neoestructuralismo y las estrategias de desarrollo en América Latina. En H. Márquez, R. Soto y E. Záyago (Coords), El desarrollo perdido. Avatares del capitalismo neoliberal en tiempos de crisis (pp.55-69). Recuperado de http://rimd.reduaz.mx/coleccion desarrollo migracion/desarrollo perdido/4.pdf

14

Shirley Benavides Vindas

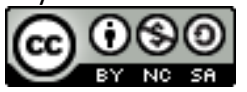

Revista Economía y Sociedad by Universidad Nacional is licensed under a CreativeCommons Reconocimiento-NoComercial- 
Lustig, N. (1987). Del estructuralismo al neoestructuralismo: la búsqueda de un paradigma heterodoxo documento de trabajo (Núm. X-1987). Recuperado del sitio del Centro de estudios económicos del Colegio de México: http://cee.colmex.mx/documentos/documentos-de-trabajo/1987/dt198710.pdf

Rodríguez, O. (2006). El estructuralismo latinoamericano. Recuperado de http://www.cepal.org/es/publicaciones/1952-estructuralismo-latinoamericano

Vázquez-Barquero, A. (2000). Desarrollo endógeno y globalización. EURE, 26(79). Recuperado de http://www.scielo.cl/scielo.php?script=sci_arttext\&pid=S0250-71612000007900003 